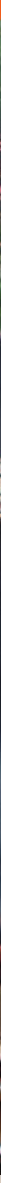

Ein Slum zwischen der Strasse und den Bahngeleisen.

«Ärzte für die Dritte Welt»: als pensionierter Arzt in Bangladesh

\title{
Humanitärer Einsatz in Dhaka
}

Balz Wolfisberg

Korrespondenz:

Balz Wolfisberg, med. pract. Facharzt FMH für Allgemeinmedizin

Wächselacher 9

CH-6370 Stans

Tel. 0416105410

balzwolfisberg[at]hotmail.com
Nach meiner Pensionierung als Hausarzt mit eigener Praxis in Stans/Nidwalden wollte ich noch etwas sinnvolles Medizinisches tun und habe mich für einen Kurzeinsatz in Dhaka, Bangladesh, gemeldet. Organisiert wurde dieser Einsatz von «Ärzte für die Dritte Welt Deutschland - German doctors».
Dhaka ist eine typische Drittweltstadt mit grosser Armut, Müllproblemen und chaotischem Verkehr. Von den 14 Millionen Einwohnern leben 1 bis 3 Millionen in menschenunwürdigen Slums. Die Armut bedingt, dass den meisten dieser Menschen der Zugang zur medizinischen Grundversorgung ver-

«Dieser erste Einsatz war für mich eine grosse persönliche und fachliche Herausforderung.»

In zwei Wochenendseminaren in Frankfurt am Main habe ich mich auf diese Aufgabe vorbereitet und vorgängig mein Gymnasialenglisch auf der Insel Malta etwas aufgebessert. Erste Drittwelterfahrungen, die ich 1980 während eines 4-monatigen Aufenthaltes in einem Bergspital in Lesotho mit Solidarmed Schweiz gemacht hatte, sollten mir zugute kommen. Mit diesem Rüstzeug flog ich Mitte August für 6 Wochen nach Dhaka. wehrt ist und viele an eigentlich heilbaren Krankheiten sterben.

Ich habe zusammen mit einem Kollegen aus Innsbruck, später einer Kollegin aus Salzburg, in einem Slum am Stadtrand gearbeitet. Meine mitgebrachten Hilfsmittel waren das Stethoskop, das Otoskop und eine gute Taschenlampe. Vor Ort war ein Team mit einer ausgebildeten Krankenschwester, zwei Übersetzern, einer Verantwortlichen für die Apotheke und 


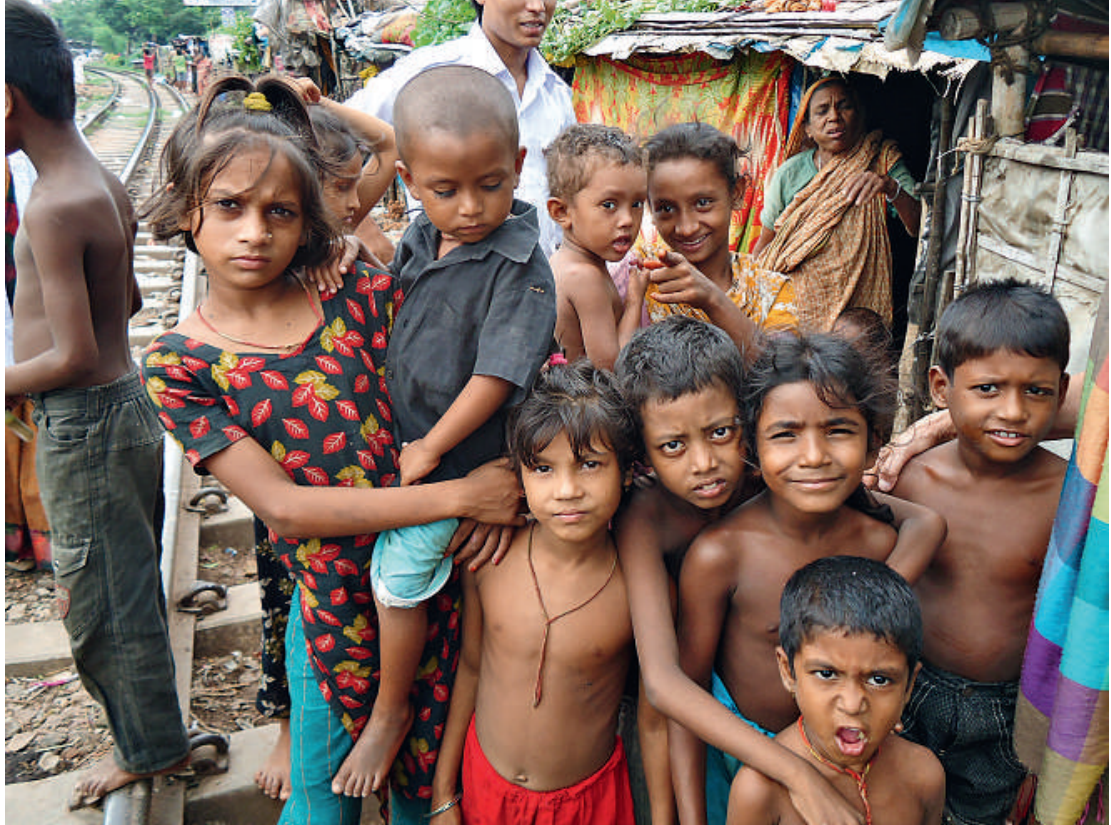

Die Kinder leben in dauernder Gefahr vor heranrollenden Zügen.

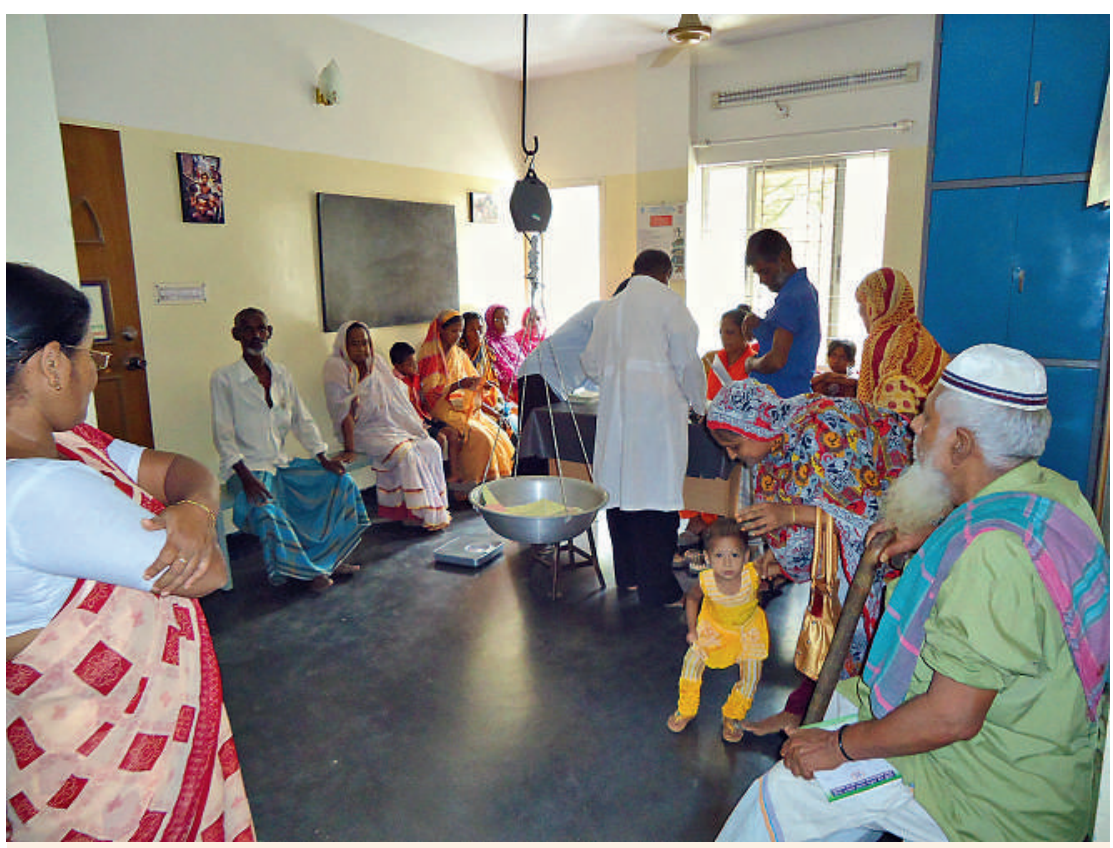

Ein Warteraum im Ambulatorium von Manda.

einem Fahrer. Am Morgen fuhren wir jeweils in andere Slums mit bis zu einer Stunde Anfahrt, einmal am Schluss mit einem Holzboot über einen See. Die meisten Slums sind an unwirtlichen Orten zwischen Strassen, an Bahngeleisen oder unter Brücken gelegen. Es passieren immer wieder schwere Unfälle mit Kindern, die von heranrollenden Zügen überfahren werden.

In die Sprechstunde kamen vorwiegend untergewichtige Frauen mit ihren unterernährten Kindern, aber auch ältere Frauen und Männer. Es galt, schwere
Leiden wie Pneumonien bei Kindern, schwere Diarrhoe, COPD, Anämien, Verdacht auf TBC und Lepra, Vitaminmangel, Abszesse und Otitis purulenta $\mathrm{zu}$ erkennen und die nötige Therapie und Abklärung in die Wege zu leiten. Viele Patienten hatten Schmerzprobleme, deren Hintergründe nicht immer eruiert und die meistens symptomatisch behandelt wurden. Von Männern geschlagene Frauen gehörten zum Sprechstundenalltag. Mit Empathie und Paracetamol konnten wir dieses Problem nicht lösen, höchstens den physischen und psychischen Schmerz etwas lindern.

Häufig waren Hautkrankheiten wie Scabies und Pyodermien. Untergewichtige Kinder bis 5 Jahre wurden in die von uns betreute Feeding-Station aufgenommen, wo die Kinder eine ausgewogene Ernährung und die Mütter entsprechende Beratung erhielten. Bei unklaren Krankheiten konnten wir die Dienste eines privaten Labors inklusive Röntgen in Anspruch nehmen oder die Patienten in ein Spital überweisen.

Die Präsenz der German doctors ist sinnvoll, da keine Ärzte von Bangladesh in den Slums arbeiten wollen. Dieser erste Einsatz war für mich eine grosse persönliche und fachliche Herausforderung. Ich bin bereichert zurückgekehrt, aber auch erleichtert, dass ich gesund blieb. Mein nächster Einsatz ist im Mai 2012 in Chittagong, ebenfalls Bangladesh, geplant. Als humanitären Dienst am kranken Menschen möchte ich solche Einsätze auch pensionierten Ärztinnen und Ärzten weiterempfehlen.

Kontakt und nähere Information: Ärzte für die Dritte Welt, Offenbacher Landstrasse 224, D-60599 Frankfurt am Main, Tel. 004969707997 0. www.aerzte3welt.de

«Ärzte für die Dritte Welt Deutschland» ist ein Verein mit Sitz in Frankfurt, der 1983 gegründet wurde. Sein Ziel ist es, in den Slums der Megastädte der Dritten Welt den Menschen eine medizinische Grundversorgung anzubieten. Zurzeit laufen verschiedene Langzeitprojekte in fünf Ländern: den Philippinen, in Indien, Bangladesh, Kenia und Nicaragua. Die Ärzte betreuen Gesundheitszentren, Tuberkulose- und Ernährungsprogramme, arbeiten in Rolling-Kliniken und unterstützen Schulen für Kinder aus den Slums. Die Einsätze dauern in der Regel sechs Wochen oder länger. Die meisten sind Allgemeinärzte, Internisten und Kinderärzte, fast ebenso viele Frauen wie Männer. Viele sind noch in der Klinik tätig, arbeiten in ihrer eigenen Praxis und opfern so ihre Ferien, oder sind bereits pensioniert.

Als gemeinnütziger Verein finanziert sich «Ärzte für die Dritte Welt» vorwiegend aus Spenden, für einzelne Projekte erhält er auch Bundesmittel. Die Ärzte im Einsatz zeigen ihre Solidarität, indem sie unentgeltlich arbeiten und die Hälfte der Reisekosten selber bezahlen. 